\title{
Die Bonebridge - das erste aktive Knochenleitungsimplantat
}

\author{
Martin Leinung, Tobias Rader, Timo Stöver
}

\section{Zusammenfassung}

Knochenleitungshörgeräte regen das Innenohr über Körperschall an, der auf unterschiedliche Arten auf den Schädelknochen übertragen werden kann. Die beste Ankopplung erhält man, wenn das Hörsystem fest mit dem Knochen verbunden wird. In der Praxis bedeutete dies bislang, dass die Patienten dauerhaft eine die Haut penetrierende und somit ständig sichtbare Titanschraube hinter dem Ohr trugen. Hierdurch besteht das Risiko einer Infektion, die schlimmstenfalls zum Verlust des Implantats führen könnte. Seit letztem Jahr ist nun erstmalig ein teilimplantierbares Knochenleitungsgerät verfügbar, das diese Nachteile umgeht.

\section{The Bonebridge - The First Active Bone Conduction Implant}

Bone conduction hearing aids stimulate the inner ear via the impact of sound waves, which can be transmitted to the cranial bone by various ways. The optimum coupling is achieved when the hearing device is tightly fixed to the bone. Therefore, the patients are equipped with a skinpenetrating and permanently visible screw behind the auricle. The patients thus bear an inherent risk of wound infection up to implant loss. In 2012 the first implantable bone conduction implant became available, which now avoids these drawbacks.

\section{Herkömmliche Knochenleitungs- hörgeräte}

Als Knochenleitung bezeichnet man die Übertragung von Schallwellen durch den Schädelknochen zum Innenohr. Dieses physiologischen Prinzips bedienen sich Hals-Nasen-Ohren-Ärzte z. B. bei den orientierenden Stimmgabeluntersuchungen des Hörvermögens nach Weber und Rinne (oder aktuell die Datenbrille „Glass“ von Google).

Seit über 30 Jahren stehen Knochenleitungshörgeräte für die Patientenversorgung zur Verfügung (z.B. BAHA = bone anchored hearing aid). Im Gegensatz zu konventionellen Hörgeräten wird das akustische Signal nicht mithilfe von miniaturisierten Lautsprechern verstärkt, sondern über ein Vibrationselement auf den Knochen übertragen. Die Ankopp-

OP-JOURNAL 2013; 29: 206-209

(c) Georg Thieme Verlag KG Stuttgart · New York DOI http://dx.doi.org/10.1055/s-0033-1350763 lung kann hierbei auf unterschiedliche Arten erfolgen: Bei Kindern mit noch nicht ausreichend ausgebildeter Schädelkalotte wird das Vibrationselement mit einem elastischen Stirnband an die Haut über dem Ohrknochen gepresst. Ferner ist die Integration in das Bügelende einer Brille möglich (Knochenleitungsbrille). Eine bessere Ankopplung an den Schädelknochen (und damit eine bessere Klangqualität und -verstärkung) wird jedoch durch ein invasives Verfahren erreicht, bei dem eine Schraube direkt in die Squama des Os temporale implantiert wird, an die der Patient das eigentliche Knochenleitungshörgerät mithilfe einer Schnappkupplung kraftschlüssig anbringen kann [1]. Bei der Operation wird ein definierter Hautdefekt angelegt, durch den die BAHASchraube dauerhaft sichtbar ist. Zur Vermeidung einer Infektion wird der Hautlappen um die Schraube herum von subkutanem Fettgewebe befreit, um eine direkte narbige Verbindung mit dem Knochen einzugehen. Die Patienten sind angehalten, täglich mit einer weichen
Bürste Verschmutzungen an der Kontaktstelle zwischen Schraube und Haut zu reinigen. Studien haben jedoch gezeigt, dass die Komplikationsrate in der Langzeitbeobachtung mit 13,6\% Implantat-Verlustrate und $16,9 \%$ schweren Hautreizungen im Schraubenbereich sehr hoch liegt [2,3].

\section{Das Knochenleitungsimplantat Bonebridge}

2012 wurde nach präklinischen Studien an humanen Felsenbeinpräparaten und nicht invasiven Tests an Normalhörenden [4] erstmalig ein transkutan aktiviertes Knochenleitungssystem auf dem Markt eingeführt. Hierbei wird das Vibrationselement im Rahmen einer einfachen Operation in das Mastoid eingebracht und mithilfe von Osteosyntheseschrauben fixiert. Die Haut über dem Implantat ist verschlossen. Die Steuerung des Implantats erfolgt durch einen auf der Haut getragenen Prozessor, der zudem 2 Mikrofone sowie die Batterie enthält (vgl. Abb.1). In der Mitte der Sende- und Implantatspule sind jeweils Magnete angebracht, wodurch der Prozessor ohne weitere Fixierungen getragen werden kann. Die Stromversorgung und Signalübermittlung zwischen Prozessor und Implantat erfolgt per Induktion. Dieses Prinzip der transkutanen Signaltransduktion hat sich bei Cochleaund Mittelohrimplantaten bereits zigtausendfach bewährt.

\section{Indikationsstellung}

Die Indikationsstellung zur Versorgung mit einer Bonebridge erfolgt durch den HNO-Arzt nach Durchführung einer Reinton- und Sprachaudiometrie. Die audiologischen Kriterien sind entweder

- eine Innenohrschwerhörigkeit nicht schlechter als $45 \mathrm{~dB}$ HL bei zusätzlicher Schallleitungsschwerhörigkeit größer $30 \mathrm{~dB}$ oder 

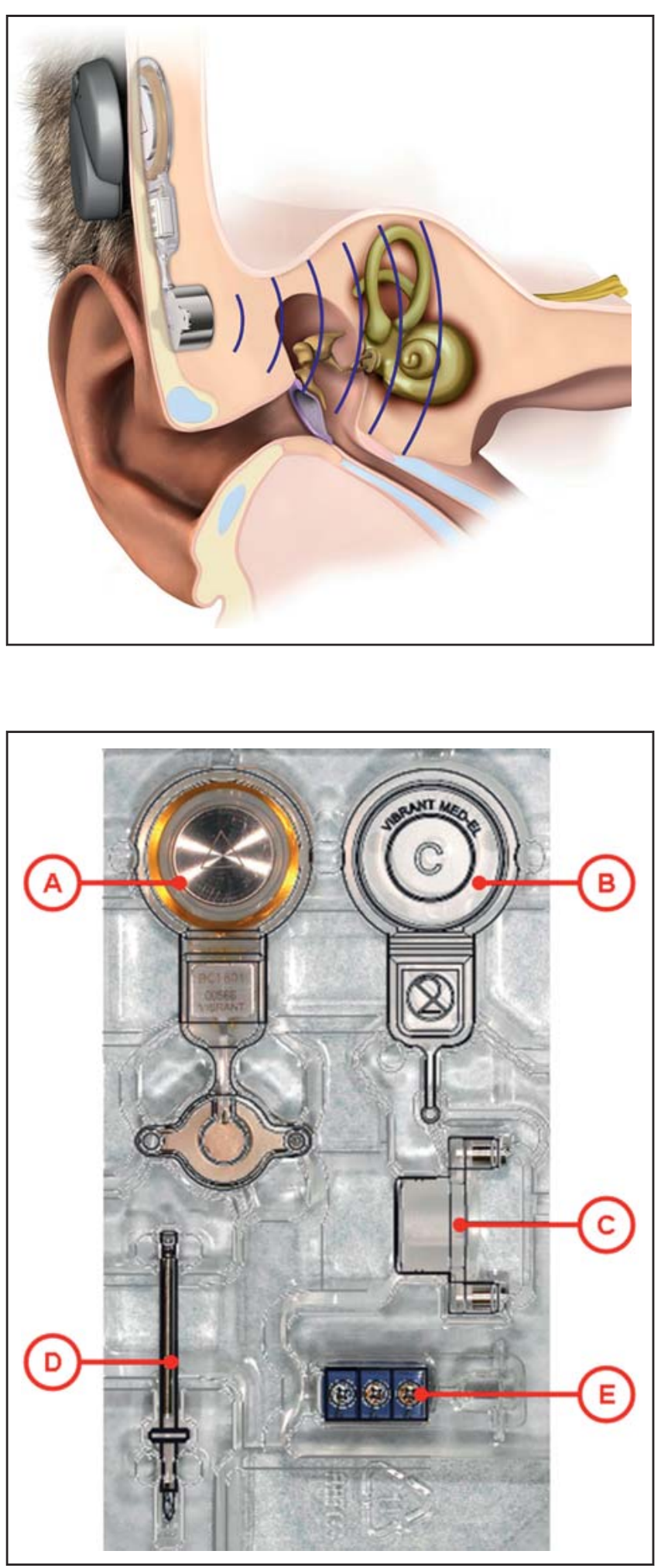

Abb. 2 Implantatset bestehend aus Bonebridge-Implantat (A), C(oil)-Sizer (B), T(ransducer)-Sizer (C), Versenkbohrer (D), $2 \times$ Titanschrauben sowie 1 Rescue-Schraube (E). Separat verfügbar sind ferner eine Messlehre zur Bestimmung der Dicke des Hautperiostlappens über dem Implantat (max. $7 \mathrm{~mm}$ für eine adäquate Signaltransduktion) sowie ein mechanischer Drehmomentschlüssel.

- eine ipsilaterale Taubheit bei kontralateraler Normakusis bzw. eine Luftleitungsschwelle nicht schlechter als $20 \mathrm{~dB}$ HL

Da die Schallübertragung per Knochenleitung nicht von der Funktionsfähigkeit des Mittelohrs abhängig ist, ist die Bonebridge eine ideale Versorgungsmöglichkeit bei operativ nicht mehr verbesserbarer Schallleitungsschwerhörigkeit, chronischer Otitis externa und media, Missbildungen des Ohres sowie Otooder Tympanosklerose, sofern jeweils die o.g. audiologischen Vorbedingungen erfüllt sind.

Eine präoperative hochauflösende Computertomografie des Felsenbeins ist obligat, um abzuschätzen, ob das Mastoid ausreichend groß für die Aufnahme des Implantats angelegt ist. Kontakt zum Sinus sigmoideus oder zur Dura ist zu vermeiden. Planungssoftware und intraoperative Navigation können die optimale Positionierung des Implantats unterstützen $[5,6]$.

\section{Operationsdurchführung}

Die bogenförmige retroaurikuläre Inzision wird nicht direkt bis auf den Schädelknochen geführt, sondern es wird ein Muskelperiostlappen präpariert, um später durch einen kulissenartigen Verschluss eine direkte Infektionsstraße vom Hautniveau bis zum Implantat zu verhindern. Anschließend wird die Oberfläche des Mastoids exponiert.

Im Implantatset sind - bis auf einen separat erhältlichen Drehmomentschlüssel und eine Messlehre für die Hautdicke alle Komponenten zur Implantation enthalten (vgl. Abb. 2). Mithilfe der Schablonen wird die Lage des Implantats festgelegt. Sowohl für den eigentlichen Aktor („Transducer“) als auch für das Implantatgehäuse (einschließlich der Empfangsspule oder „Coil“ gibt es je eine Schablone („T“- und „C-Sizer“), die miteinander verbunden werden können (Abb. 3 links). Nun wird mit Rosenbohrern absteigender Größe die zylindrische Kavität angelegt. Hierbei ist ein zu weitreichender Knochenabtrag unbedingt zu vermeiden, damit die Fixierungsschrauben beidseits des Implantatkörpers ausreichend Halt finden.

Mit dem Raspatorium nach Plester wird in direktem Knochenkontakt eine subperiostale Tasche zur späteren Aufnahme des Implantatgehäuses gebildet. Eine darüber hinausgehende Fixierung des Implantatgehäuses ist - im Gegensatz zu den Mittel- und Innenohrimplantaten aufgrund der steiferen Kabelverbindung zum Gehäuse und der Fixierung des Aktors im Knochen nicht erforderlich. Auch wäre eine Versenkung des Gehäuses in die Kalotte, wie sie z. B. bei Cochlea-Implantaten durchgeführt wird, in diesem Fall kontraproduktiv, da dadurch auch der Transducer tiefer eingesenkt werden müsste und in Kontakt mit dem Sinus sigmoideus und/oder der Dura kommen könnte. 


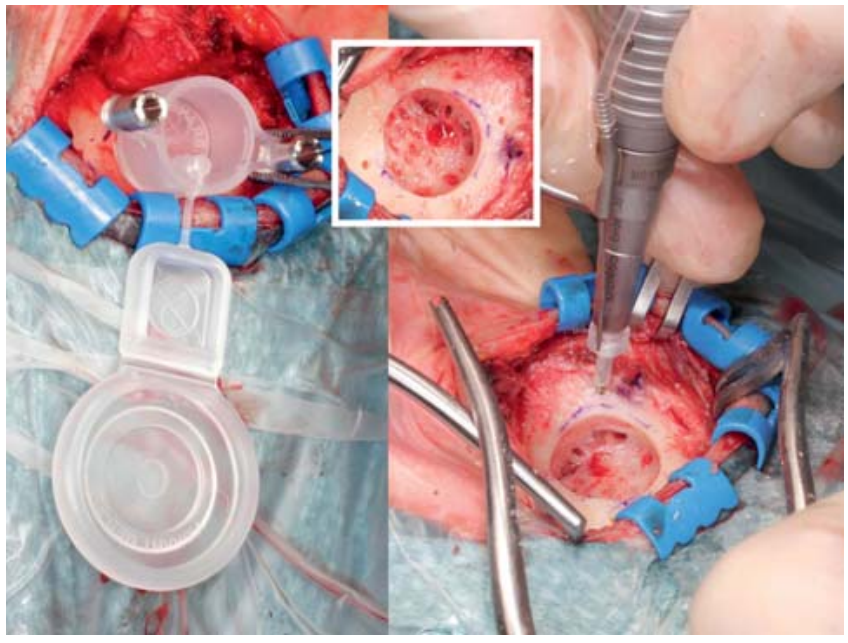

Abb. 3 Positionierung und Bohrung der Kavität zur Aufnahme des Vibrationselements. Links: Vorpositionierung mithilfe des C- und T-Sizers. Kleines Teilbild: Ausbohren der zylindrischen Kavität mit Rosenbohrern absteigender Größe. Rechts: Anlegen der Haltebohrungen mit dem Versenkbohrer.

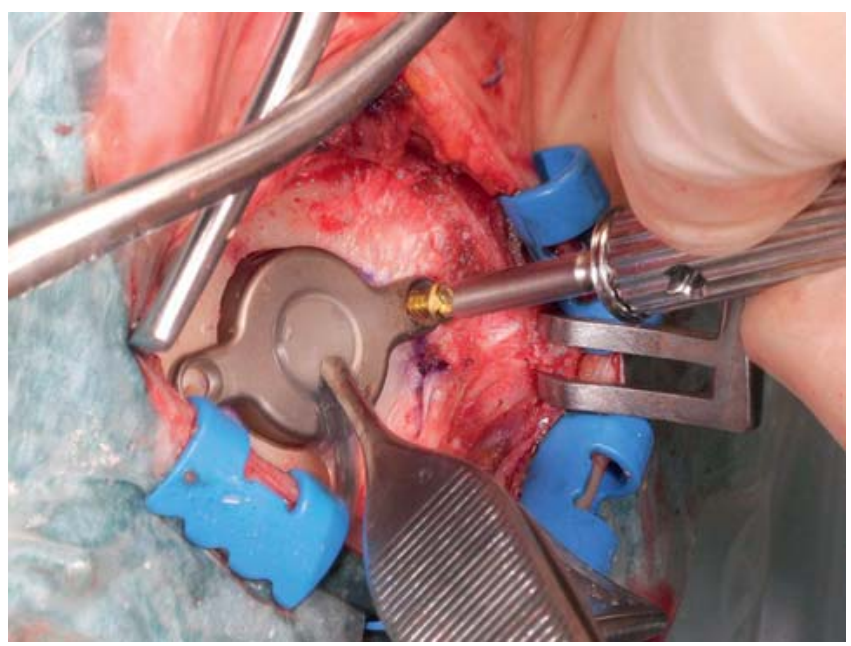

Abb. 5 Nach Platzierung des Transducers in die Kavität Befestigung mithilfe der 2 Titanschrauben, die zunächst per Hand locker angezogen werden.

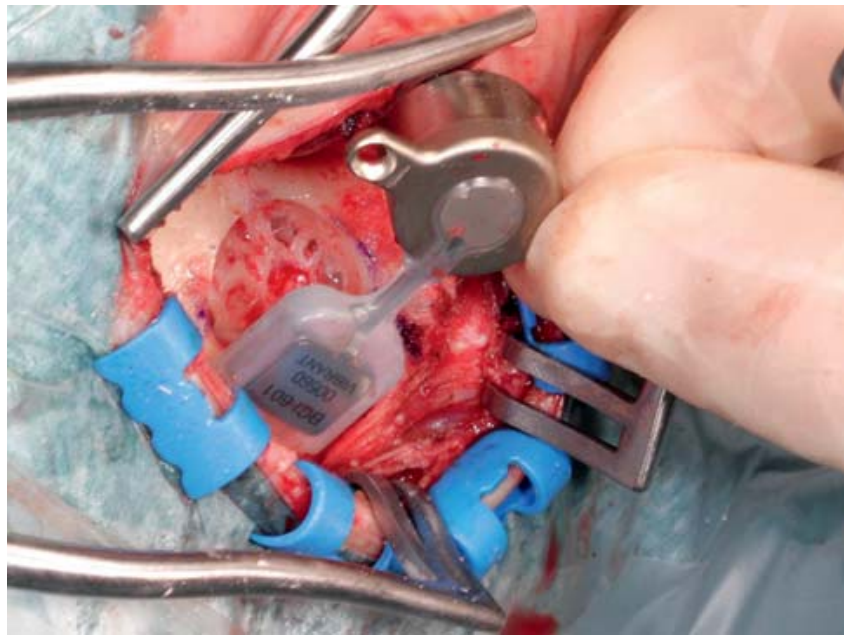

Abb. 4 Einsetzen des Implantats in die Periosttasche. Das Verbindungsstück zwischen Gehäuse und Schwinger wird anschließend vorsichtig zurechtgebogen.

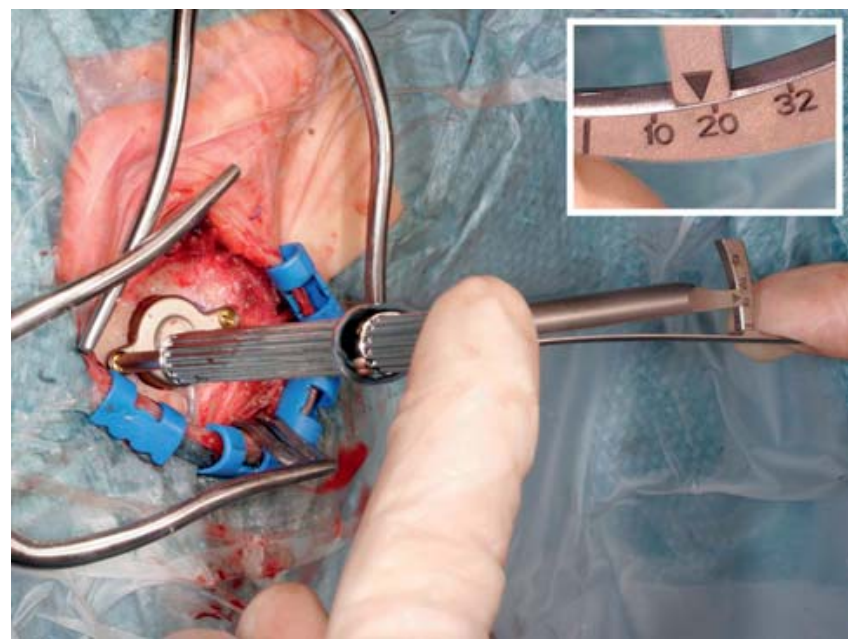

Abb. 6 Abschließend Vollendung der kraftschlüssigen Verbindung zwischen Implantat und Knochen durch Festziehen der Schrauben auf $10-20 \mathrm{Ncm}$.
Mit dem Versenkbohrer mit integriertem Distanzstück werden unter Zuhilfenahme des C-Sizers die $3,9 \mathrm{~mm}$ tiefen Bohrungen (1,4 mm Durchmesser) für die Halteschrauben ausgeführt (vgl. Abb. 3 rechts). Nun wird das Implantat in die Periosttasche geschoben und der Aktor in die Kavität eingesetzt (Abb.4). Das flexible Verbindungsstück zwischen Gehäuse und Schwinger kann vorsichtig in die endgültige Position gebogen werden. Mit 2 Osteosyntheseschrauben (Länge $6 \mathrm{~mm}$, Durchmesser $2 \mathrm{~mm}$ ) wird das Implantat beidseitig fixiert (Abb. 5). Im Set befindet sich weiterhin eine Rescue-Schraube mit einem Durchmesser von $2,4 \mathrm{~mm}$, um einen sicheren Halt des
Implantats zu gewährleisten, sofern die übliche Knochenschraube in dem Bohrloch nicht greifen sollte. Zunächst werden die Schrauben händisch locker eingedreht und anschließend mit dem Drehmomentschlüssel auf ca. 10-20 Ncm angezogen (Abb.6). Hierdurch wird eine ausreichend kraftschlüssige Fixierung des Implantats erreicht. Nach 3-schichtigem Wundverschluss (Naht des FischLappens, Subkutan- und Hautnaht) sowie Anlage eines Kompressionsverbands ist der Eingriff beendet. Das postoperative Prozedere umfasst eine bereits perioperativ begonnene Breitbandantibiose, die für weitere 4 Tage fortgeführt wird, tägliche Verbandswechsel, insbesondere mit Kontrolle einer etwaigen Serombildung über dem Implantatgehäuse, Entlassung i.d.R. am 2. oder 3. postoperativen Tag sowie Fädenentfernung am 7.10. postoperativen Tag. Nach einer ca. 4-wöchigen Einheilungsphase kann der externe Prozessor erstmalig aufgesetzt und durch einen in das System eingewiesenen Audiologen in einem mehrstufigen Anpassungsprozess eingestellt werden.

\section{Schlussfolgerung}

Das Bonebridge-Implantat ist eine wertvolle Ergänzung des bestehenden Repertoires implantierbarer Hörsysteme. Es 
kombiniert das physiologische Prinzip der Knochenleitung mit dem bewährten Konzept der transkutanen Signaltransduktion zwischen einem vollimplantierten Stimulator und einem äußerlich getragenen Prozessor. Die Erfahrungen der in unserer Klinik bislang versorgten 9 Patienten sowie die ersten publizierten Case Reports sind durchweg positiv; es sind derzeit keine Komplikationen bekannt [7-9].

\section{Literatur}

${ }^{1}$ Christensen L, Smith-Olinde L, Kimberlain J et al. Comparison of traditional bone-conduction hearing AIDS with the Baha system. J Am Acad Audiol 2010; 21: 267-273

2 Tjellström A, Stalfors J. Bone-anchored hearing device surgery: A 3- to 6-year follow-up with life table and worst-case scenario calculation. Otol Neurotol 2012; 33: 891-894

${ }^{3}$ de Wolf MJ, Hol MK, Mylanus EA et al. Boneanchored hearing aid surgery in older adults: implant loss and skin reactions. Ann Otol Rhinol Laryngol 2009; 118: 525-531

${ }^{4}$ Huber AM, Sim JH, Xie YZ et al. The Bonebridge: Preclinical evaluation of a new transcutaneously-activated bone anchored hearing device. Hear Res 2013; 301: 93-99

${ }^{5}$ Lassaletta L, Sanchez-Cuadrado I, Muñoz E et al. Retrosigmoid implantation of an active bone conduction stimulator in a patient with chronic otitis media. Auris Nasus Larynx 2013; DOI: 10.1016/j.anl.2013.04.004

${ }^{6}$ Canis M, Ihler F, Blum J et al. CT-gestützte Navigation zur retrosigmoidalen Implantation der Bonebridge. HNO 2013; DOI: 10.1007/ s00106-012-2652-5

7 Sprinzl G, Lenarz T, Ernst A et al. First european multicenter results with a new transcutaneous bone conduction hearing implant system: short-term safety and efficacy. Otol Neurotol 2013; 34: 1076-1083

8 Clarós P, Diouf M, Clarós A. Mise en place d'un "Bonebridge ${ }^{\circledR}$ ". Rev Laryngol Oto Rhinol 2012: 133: 217-220

9 Barbara M, Perotti M, Gioia B et al. Transcutaneous bone-conduction hearing device: audiological and surgical aspects in a first series of patients with mixed hearing loss. Acta Otolaryngol 2013; DOI: 10.3109/00016489. 2013.799293
Dr. med. Martin Leinung Stellvertretender Klinikdirektor

Dr.-Ing. Tobias Rader

Stellvertretender Leiter des Schwerpunktes Audiologische Akustik

Prof. Dr. med. Timo Stöver

Klinikdirektor

Klinik für Hals-Nasen-

Ohrenheilkunde

Universitätsklinikum der

Goethe-Universität Frankfurt am Main Theodor-Stern-Kai 7

60590 Frankfurt am Main

martin.leinung@kgu.de 